\title{
A Global Environment Analysis and Visualization System with Semantic Computing for "Multi- Dimensional World Map"
}

\author{
Yasushi Kiyoki \\ Graduate School of Media and Governance, Keio University, SFC, 5322 Endo, Fujisawa, Kanagawa, 252, \\ Japan \\ E-mail: kiyoki@sfc.keio.ac.jp
}

\begin{abstract}
Humankind, the dominant species on Earth, faces the most essential and indispensable mission; we must endeavor on a global scale to perpetually restore and improve our natural and social environments. The essential computation in environmental study is context-dependent-differential computation to analyze the changes of various situations (temperature, color, $\mathrm{CO} 2$, places of livings, sea level, coral area, etc.). It is important to realize global environmental computing methodology for analyzing difference and diversity of nature and livings in a context dependent way with a large amount of information resources in terms of global environments. It is also significant to memorize those situations and compute environment change in various aspects and contexts, in order to discover what is happening in the nature of our planet. We have various (almost infinite) aspects and contexts in environmental changes in our planet, and it is essential to realize a new analyzer for computing differences in those situations for discovering actual aspects and contexts existing in the nature. We propose a new method for Differential Computing in our Multi-dimensional World map. We utilize a multi-dimensional computing model, the Mathematical Model of Meaning (MMM), and a multi-dimensional space filtering method with an adaptive axis adjustment mechanism to implement differential computing. Computing environmental changes in multi-aspects and contexts using differential computing, important factors that change natural environment are highlighted. We also present a method to analyze and visualize the highlighted factors using our Multi-dimensional World Map (5-Dimensional World Map) System. We also introduce the concept of "SPA (Sensing, Processing and Analytical Actuation Functions)" for realizing a global environmental system, to apply it to Multi-dimensional World Map (5-Dimensional World Map) System. This concept is effective and advantageous to design environmental systems with Physical-Cyber integration to detect environmental phenomena as real data resources in a physical-space (real space), map them to cyber-space to make analytical and semantic computing, and actuate the analytically computed results to the real space with visualization for expressing environmental phenomena, causalities and influences.
\end{abstract}

Keywords: Global environmental analysis, semantic computing, multimedia system, data mining.

ENGINEERING JOURNAL Volume 20 Issue 4

Received 3 March 2016

Accepted 13 June 2016

Published 1 August 2016

Online at http://www.engj.org/

DOI:10.4186/ej.2016.20.4.137 


\section{Introduction}

As an important global environmental system, we have proposed a multi-dimensional data mining and visualization system. In the design of this global environment-analysis system, we focus on how to search and analyze environmental media data (images, sound, movies and documents) related to environmental situations, according to user's contexts and analysis-points. We have introduced a semantic associative computing system based on our "MMM: the Mathematical Model of Meaning" [1 - 3]. This system realizes semantic associative computing and search for media data and it is applied to compute semantic correlations between keywords, images, sensing data, sound data and documents dynamically in a contextdependent way. The main feature of this system is to realize semantic associative search and analysis in the 2000 dimensional orthogonal semantic space with semantic projection functions. This space is created for dynamically computing semantic equivalence or similarity between keywords and media data.

\section{Global Environmental Analysis with Semantic Computing}

One of the important applications of the semantic associative computing system is "Global EnvironmentAnalysis," as shown in Fig. 1, which aims to evaluate various influences caused by natural disasters in global environments. Our experimental results have shown the feasibility and effectiveness of our semantic associative computing system based on "MMM" in global environmental analysis. We have constructed "3D Cyber Space System for Environmental Analysis," as shown in Fig. 2, for sharing and analyzing environmental situations with MMM functions applied to "environmental multimedia data sharing," as a new platform of collaborative environment analysis [4, 6]. This platform enables to create a remote, interactive and real-time environmental and academic research exchange among different areas.

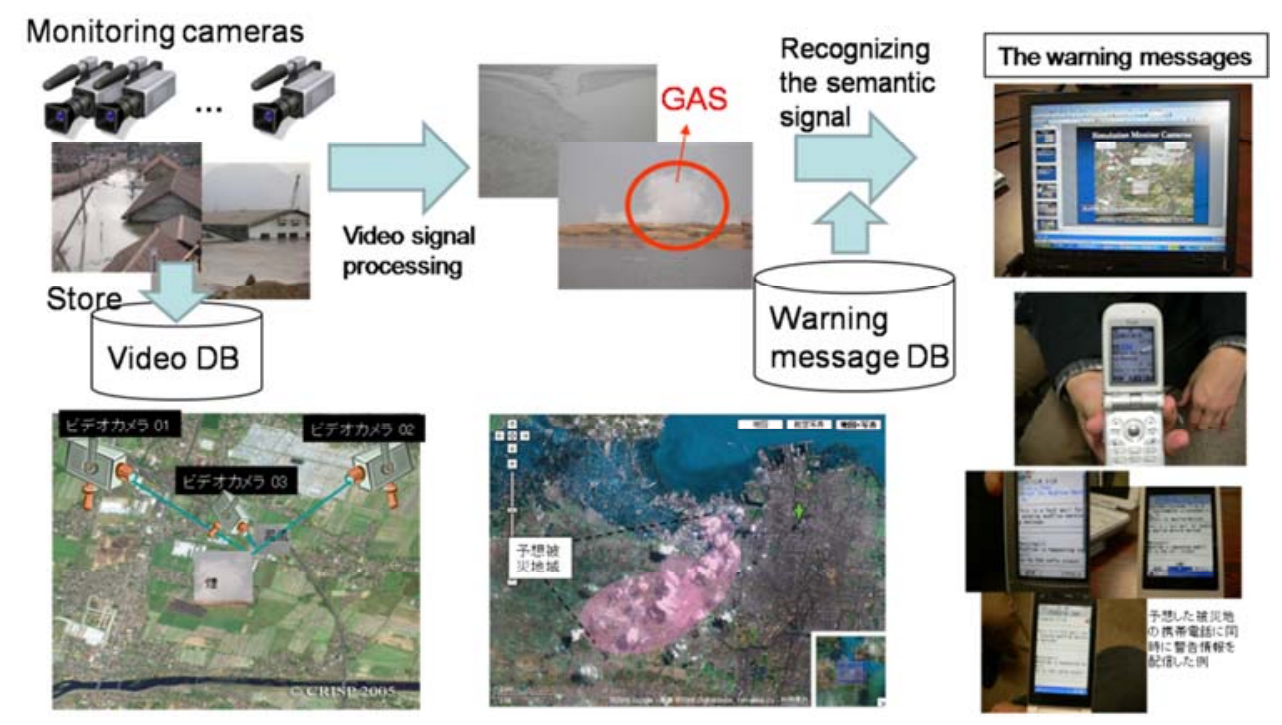

Fig. 1. Environmental analysis with the semantic associative computing system. 


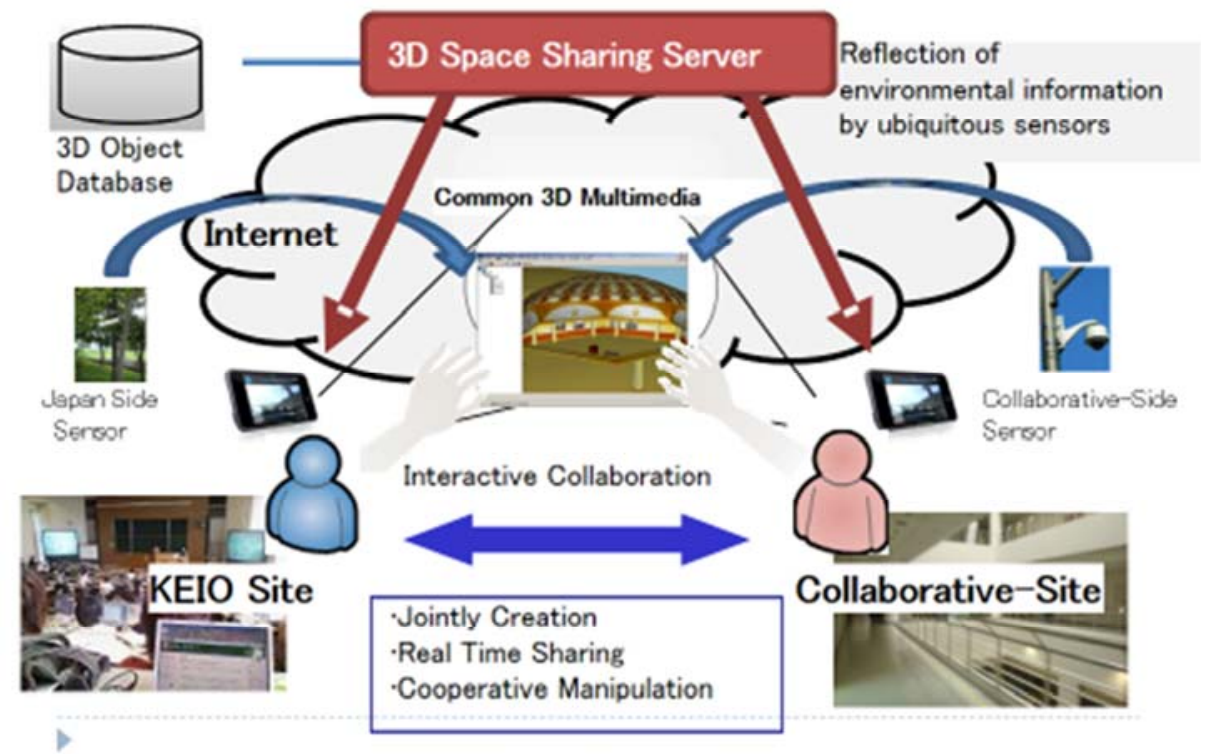

Fig. 2. 3D cyber space system for environmental analysis in remote areas.

\subsection{Semantic Computing in MMM}

In the Mathematical Model of Meaning (MMM) [1, 2, 7], an orthogonal semantic space is created for semantic associative search. Retrieval candidates and queries are mapped onto the semantic space. The semantic associative search is performed by calculating the correlation of the retrieval semantic space.

In MMM, the acquisition of information or knowledge is performed by semantic computations. Context-dependent interpretation means that information is dynamically extracted by a semantic computation with context-recognition. The method realizes the computational machinery for recognizing the meaning of contexts and obtaining the semantically related information to the given context. MMM is essentially different from those methods. The essential difference is that this method provides dynamic recognition of the context. That is, the "context-dependent interpretation" is realized by dynamically selecting a certain subspace from the entire semantic space. The other methods do not provide the context dependent interpretation, that is, their space is fixed and static. The outline of MMM $[1,2,7]$ is summarized as the following:

(1) A set of $m$ words is given, and each word is characterized by $n$ features. That is, an $m$ by $n$ matrix $\boldsymbol{M}$ is given as the data matrix.

(2) The correlation matrix $\boldsymbol{M}^{\boldsymbol{T}} \boldsymbol{M}$ with respect to the $n$ features is constructed from the matrix $\boldsymbol{M}$. Then, the eigenvalue decomposition of the correlation matrix is computed and the eigenvectors are normalized. The orthogonal semantic space $\boldsymbol{M D S}$ is created as the span of the eigenvectors which correspond to nonzero eigenvalues.

(3) "Context words" and "image" are characterized as "context" by using the $n$ features and representing them as $n$-dimensional vectors.

(4) The context words and "image" are mapped into the orthogonal semantic space by computing the Fourier expansion for the $n$-dimensional vectors.

(5) A set of all the projections from the orthogonal semantic space to the invariant subspaces (eigen spaces) is defined. Each subspace represents a phase of meaning, and it corresponds to "context."

(6) A subspace of the orthogonal semantic space is selected according to the given "context" expressed in $n$-dimensional vectors, which are given as "context" represented by "a sequence of words" and "image."

(7) The most correlated information resources to the given "context" are extracted as the selected subspace by applying the metric defined in the semantic space.

\subsection{The Fundamental Aspects of MMM}

The advantages and original points of this method are summarized as follows: 
(1) The semantic associative media search based on semantic computation is realized by a mathematical approach. This media search method surpasses the search methods which use pattern matching for associative search. Users can use their own words or images for representing impression and data contents for media retrieval, and do not need to know how the metadata of media data of retrieval candidates are characterized in databases.

(2) Dynamic context recognition is realized using a mathematical foundation. The context recognition can be used for obtaining multimedia information by giving the user's impression and the contents of the information as "context". A semantic space is created as a space for representing various contexts which correspond to its subspaces. A context is recognized by the computation for selecting a subspace.

The essential advantage is that this method provides the important function for semantic projections which realizes the dynamic recognition of "context". That is, the "context-dependent interpretation" is dynamically performed by computing the distance between different media data, information resources and words in a context-dependent way. The context-dependency is realized by dynamically selecting a subspace from the entire orthogonal semantic space, according to "context". In this method, the number of phases of contexts is almost infinite (currently $2^{2000}$ in the general English word space [5] and $2^{180}$ in the colorimage space, approximately). For semantic associative computations of "Kansei" information [9], this method constructed several actual semantic spaces, such as the general English-word space in 2115 dimensions, the color-image space in 183 dimensions, and music space in 8 dimensions in the current implementations.

\section{Spatio-Temporal and Semantic Computing}

We have introduced the architecture of a multi-visualized and dynamic knowledge representation system "5D World Map System [4, 6, 8]," applied to environmental analysis and semantic computing. The basic space of this system consists of a temporal (1 $1^{\text {st }}$ dimension), spatial ( $2^{\text {nd }}, 3^{\text {td }}$ and $4^{\text {th }}$ dimensions $)$ and semantic dimensions $\left(5^{\text {th }}\right.$ dimension, representing a large-scale and multiple-dimensional semantic space that is based on our semantic associative computing system (MMM). This space memorizes and recalls various multimedia information resources with temporal, spatial and semantic correlation computing functions, and realizes a 5D World Map for dynamically creating temporal-spatial and semantic multiple views applied for various "environmental multimedia information resources."

\subsection{Semantic Computing in 5D World Map System}

We apply the dynamic evaluation and mapping functions of multiple views of temporal-spatial metrics, and integrate the results of semantic evaluation to analyze environmental multimedia information resources. MMM is applied as a semantic associative search method [1 - 4] for realizing the concept that "semantics" and "impressions" of environmental multimedia information resources, according to the "context". The main feature of this system is to create world-wide global maps and views of environmental situations expressed in multimedia information resources (image, sound, text and video) dynamically, according to user's viewpoints. Spatially, temporally, semantically and impressionably evaluated and analyzed environmental multimedia information resources are mapped onto a 5D time-series multi-geographical space. The basic concept of the 5D World Map System is shown in Figures 3 and 4. The 5D World Map system applied to environmental multimedia computing visualizes world-wide and global relations among different areas and times in environmental aspects, by using dynamic mapping functions with temporal, spatial, semantic and impression-based computations $[5,6,7,8]$.

\subsection{SPA: Sensing, Processing and Analytical Actuation Functions in 5D World Map}

"SPA" is a fundamental concept for realizing environmental system with three basic functions of "Sensing, Processing and Analytical Actuation" to design a global environmental system with Physical-Cyber integration. "SPA" is effective and advantageous to detect environmental phenomena as real data resources in a physical-space (real space), map them to cyber-space to make analytical and semantic computing, and actuate the analytically computed results to the real space by visualization for expressing environmental phenomena with causalities and influence. This concept is applied to our semantic computing in 5D World Map System, as shown in Figs. 5 and 6. 


\section{The concept of 5D World Map}

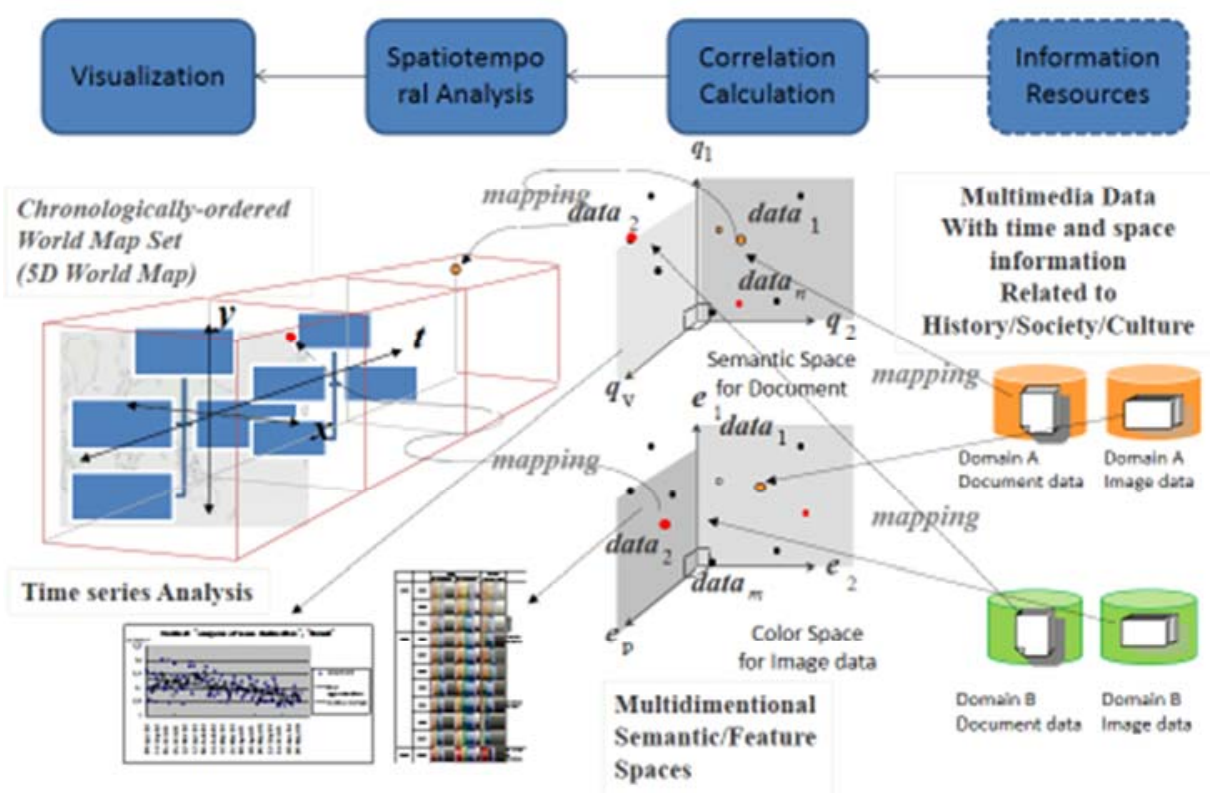

Fig. 3. 5D world map system for world-wide viewing for global environmental analysis.

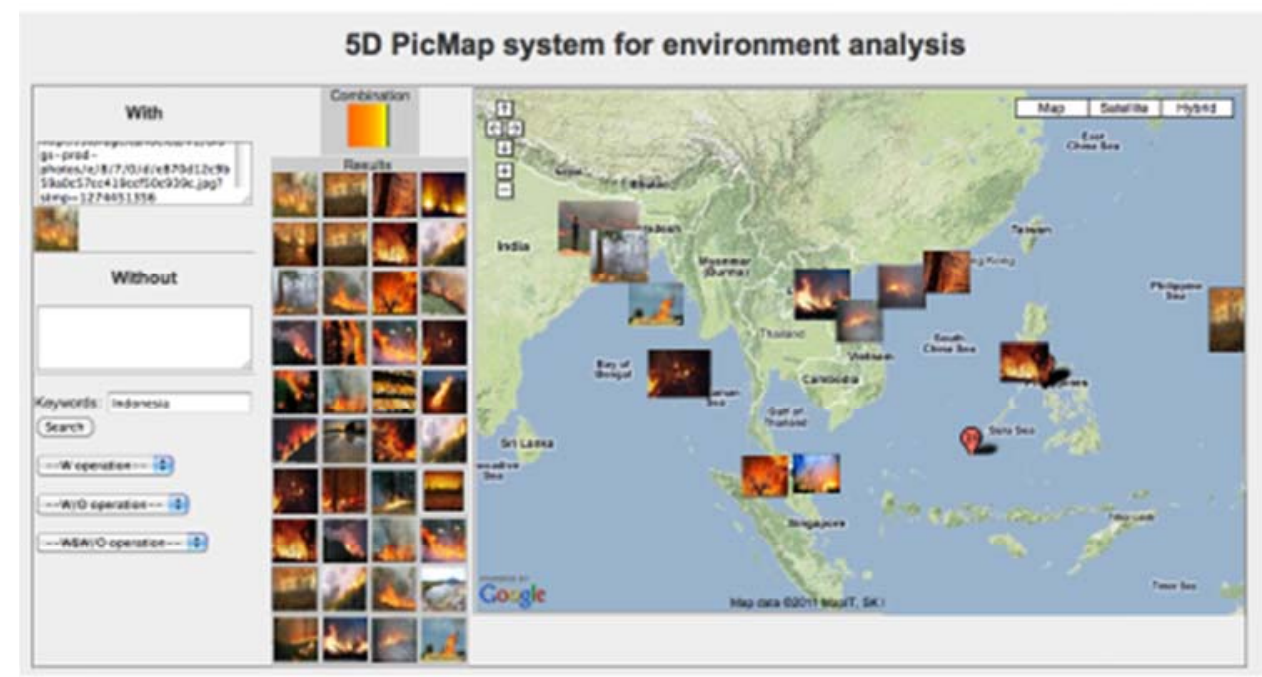

Fig. 4. Global environmental analysis of "forest fire" in 5D world map system. 


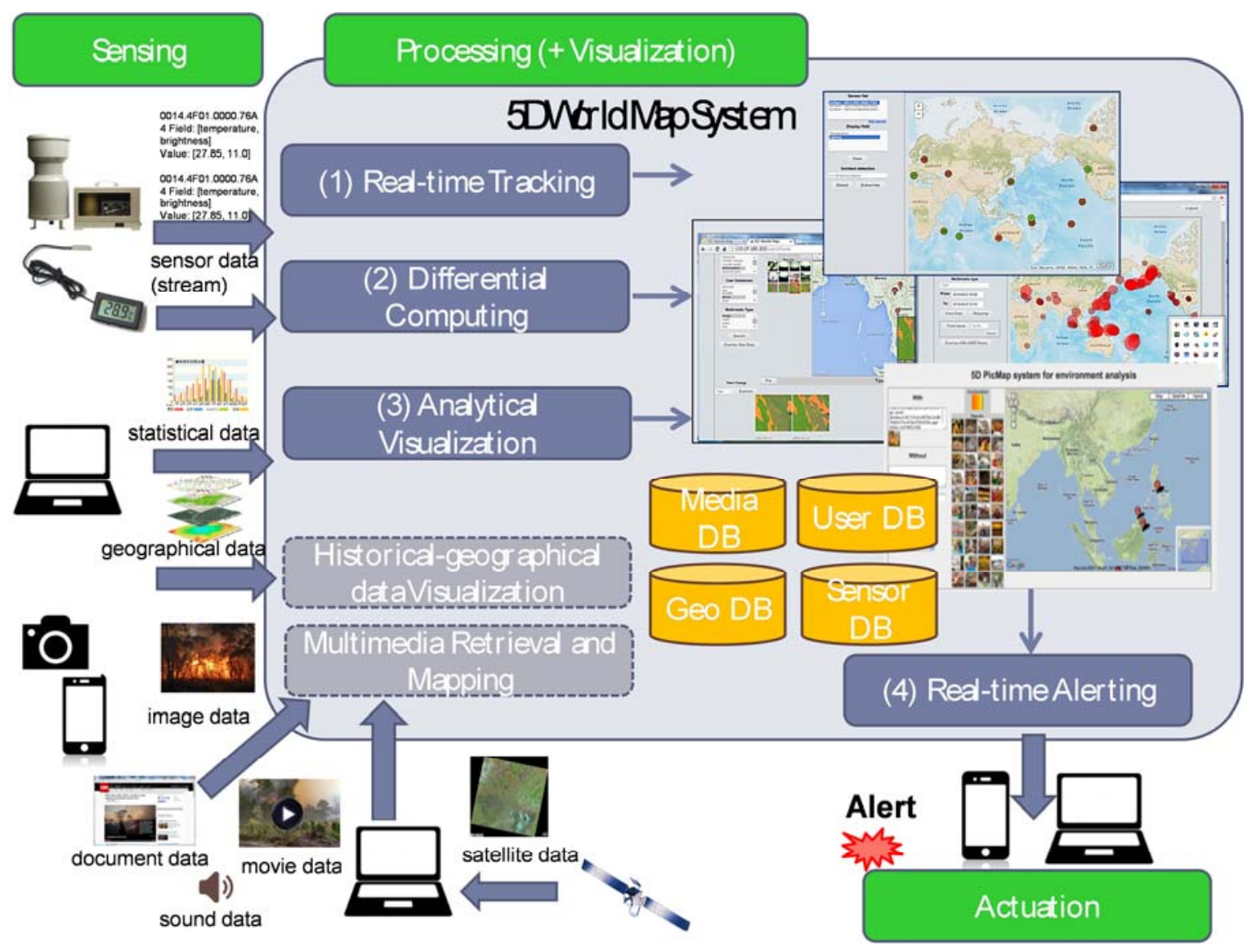

Fig. 5. Basic SPA functions in 5D world map system.

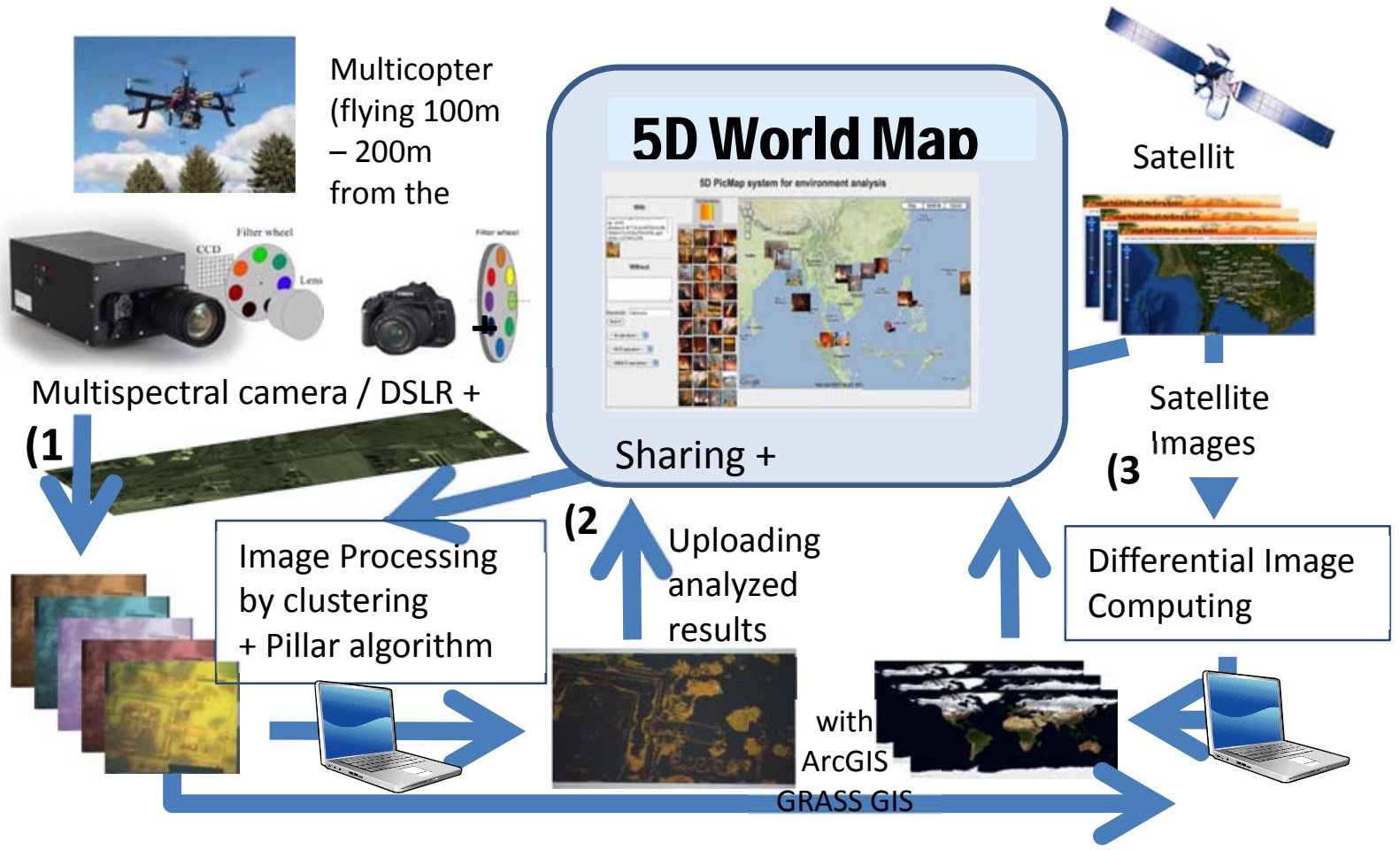

Fig. 6. SPA functions for multispectral environment-images in 5D world map system. 


\section{4. "Multi-Spectral Semantic-Image Space"}

In this section, we introduce a "multi-spectrum semantic-image space" in 6 dimensions ((a) IR cut-off filtered axis, (b) Blue filtered axis, (c) Blue-Green filtered axis, (d) Green filtered axis, (e) Red filtered axis, and (f) InfraRed pass filtered axis). The brightness in the corresponding pixel in each filtered image is mapped as the value of the corresponding axis. For example, when "Spot-S" in some place is mapped into 6-dimensional multi-spectrum image space, the brightness of the pixel- $\mathrm{A}$ in the axis is defined as a value of Spot- $\mathrm{A}$ in this axis. That is, six values are defines for Spot-S in six axes in this semantic space, and we can apply semantic computing in MMM to the multi-spectrum images. This semantic space and its implementation are introduced in $[8]$ in detail.

The 6-dimentional "multi-spectrum semantic-image space" consists of 6 axes, each of which corresponds to a single spectrum image filtered by a spectrum (color) filter.

(Axis-A) IR cut-off filtered axis: This axis is used as similar to human eye, because we use a common Digital single lens reflex (DSLR) camera in which IR cut filter is removed to detect IR spectra using IR pass filter. That is, the camera's IR cutting function (the sensor to cut off the IR spectra) is customized by expanding its wavelength responses to the IR region to acquire multispectral images.

(Axis-B) Blue filtered axis: With Blue filter: wave length $0.45-0.515 \mu \mathrm{m}$ (Visible): this axis of the spectrum is used to analyse the characteristics of the water, the land-use, the soil and the plants. This part is easy to be effected by the air diffusion.

(Axis-C) Blue-Green filtered axis: With Blue-Green filter: wave length 0.515-0.525 $\mu \mathrm{m}$ (Visible): this axis of the spectrum is also used to analyse the characteristics of the water, the land-use, the soil and the plants. The leaves turn orange and look bright, while the water turns cyan and looks darker by this filter.

(Axis-D) Green filtered axis: With Green filter: wave length 0.52-0.60 $\mu \mathrm{m}$ (Visible): this axis of the spectrum reflects the chlorophyll of the plants with leaves in the color between blue to red. This filter is useful to analysis the plant's activity.

(Axis-E) Red filtered axis: With Red filter: wave length 0.63-0.69 $\mu \mathrm{m}$ (Visible): this axis reflects the chlorophyll of the plants with green leaves. This axis is the most important for vegetation analysis, and extraction of the waterline and the differentiation of the borderline of the different soil's nature - dry or wet.

(Axis-F) Infra-Red pass filtered axis: With IR pass filter: wave length 0.76-0.90 $\mu \mathrm{m}$ (Invisible): this axis of the spectrum is especially important for ecology because healthy plants reflect it - the water in their leaves scatters the wavelengths back into the sky. By comparing it with other bands, we get indexes, which let us measure plant health more precisely than if we only looked at visible greenness. The leaves are brighter than the normal image, while the water is darker by this filter. This axis corresponds to biomass. This axis is useful to distinguish the land and the water area.

We have realized the "multi-spectrum semantic-image space" in 6 dimensions in the 5D World Map system. The brightness in the corresponding pixel in each filtered image is mapped as the value of the corresponding axis ((a) IR cut-off filtered axis, (b) Blue filtered axis, (c) Green filtered axis, (d) Red filtered axis, and (e) Infra-Red pass filtered axis (IR76)).

\section{Conclusion}

We have presented a new concept of "Multimedia Computing System" for realizing global environmental analysis and communication. The main feature of this system is to realize semantic associative search in the 2000 dimensional orthogonal semantic space with semantic projection functions. This space is created for dynamically computing semantic equivalence or similarity between keywords and media data.

We have applied this system to "global environmental system research and education" as a new platform of environmental computing. This system enables to create a remote, interactive and real-time environmental and academic research exchange between remote areas. We have also presented Environmental Multimedia Computing system with the 3D Cyber Space System, and the 5D World Map System, as an international and environmental research platform with Spatio-temporal and semantic analysers. We have also presented "SPA" concept and several new approaches to global environmentalanalysis and visualization by the multimedia system architecture with "spatio-temporal \& semantic computing." 
As our future work, we will extend our multimedia computing system to new international and collaborative research and education for realizing mutual understanding and knowledge sharing on global environmental issues in the world-wide scope.

\section{Acknowledgement}

The author would like to appreciate Dr. Xing Chen, Dr. Shiori Sasaki, Dr. Chawan Koopipat and Dr. Petchporn Chawakitchareon for their significant discussions and experimental studies.

\section{References}

[1] Y. Kiyoki, T. Kitagawa, and T. Hayama, "A metadatabase system for semantic image search by a mathematical model of meaning," ACM SIGMOD Record, vol. 23, no. 4, pp. 34-41, 1994.

[2] Y. Kiyoki, T. Kitagawa, and T. Hayama, "A metadatabase system for semantic image search by a mathematical model of meaning," Multimedia Data Management - Using metadata to integrate and apply digital media, McGrawHill, A. Sheth and W. Klas (editors), Chapter 7, 1998.

[3] Y. Kiyoki, and S. Ishihara, "A semantic search space integration method for meta-level knowledge acquisition from heterogeneous databases," Information Modeling and Knowledge Bases (IOS Press), vol. 14, pp. 86-103, May 2002.

[4] Y. Kiyoki, S. Sasaki, N. N. Trang, and N. T. N. Diep, "Cross-cultural multimedia computing with impression-based semantic spaces," Conceptual Modelling and Its Theoretical Foundations, Lecture Notes in Computer Science, Springer, pp. 316-328, March 2012.

[5] Y. Kiyoki, and A Kansei, "Multimedia computing system for environmental analysis and cross-cultural communication," 7th IEEE International Conference on Semantic Computing, keynote speech, Sept. 2013.

[6] S. Sasaki, Y. Takahashi, and Y. Kiyoki, "The 4D world map system with semantic and spatiotemporal analyzers," Information Modelling and Knowledge Bases, vol. XXI, IOS Press, 18 pages, 2010.

[7] T. Suhardijanto, Y. Kiyoki, and A. R. Barakbah, "A term-based cross-cultural computing system for cultural semantics analysis with phonological-semantic vector spaces," Information Modelling and Knowledge Bases, vol. XXIII, pp. 20-38, IOS Press, 2012.

[8] Y. Kiyoki, X. Chen, S. Sasaki, and C. Koopipat, "Multi-dimensional semantic computing with spatialtemporal and semantic axes for multi-spectrum images in environment analysis," Information Modelling and Knowledge Bases (IOS Press), vol. XXVI, 20 pages, March 2016. 